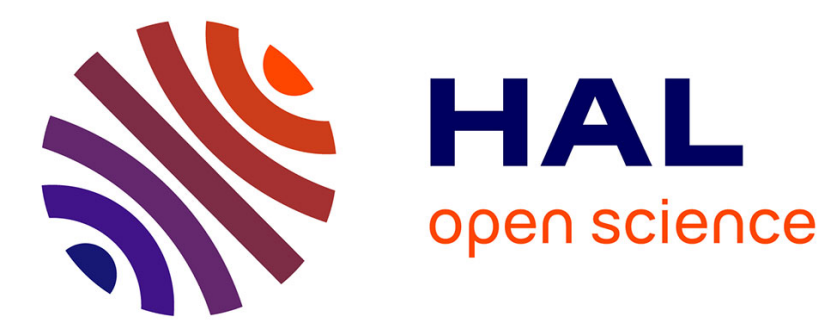

\title{
La résistance du piment (Capsicum annuum) à Phytophthora capsici. IX. Distribution spatio-temporelle du capsidiol dans les tiges infectées
}

Paul-Michel Molot, Pierre Mas, Pierre Ricci

\section{- To cite this version:}

Paul-Michel Molot, Pierre Mas, Pierre Ricci. La résistance du piment (Capsicum annuum) à Phytophthora capsici. IX. Distribution spatio-temporelle du capsidiol dans les tiges infectées. Agronomie, 1982, 2 (9), pp.865-869. 10.1051/agro:19820911 . hal-02727320

HAL Id: hal-02727320

https://hal.inrae.fr/hal-02727320

Submitted on 2 Jun 2020

HAL is a multi-disciplinary open access archive for the deposit and dissemination of scientific research documents, whether they are published or not. The documents may come from teaching and research institutions in France or abroad, or from public or private research centers.
L'archive ouverte pluridisciplinaire HAL, est destinée au dépôt et à la diffusion de documents scientifiques de niveau recherche, publiés ou non, émanant des établissements d'enseignement et de recherche français ou étrangers, des laboratoires publics ou privés. 


\title{
La résistance du piment (Capsicum annuum) à Phytophthora capsici. IX. Distribution spatio-temporelle du capsidiol dans les tiges infectées
}

\author{
Paul Michel MOLOT, Pierre MAS \& Pierre RICCI $\left(^{*}\right)$ \\ I.N.R.A., Station de Pathologie végétale, Centre de Recherches d'Avignon, F 84140 Montfavet. \\ (*) I.N.R.A., Station de Pathologie végétale, B.P. 78, F06602 Antibes.
}

\section{RÉSUMÉ}

Capsidiol, Phytoalexine, Capsicum annuum, Phytophthora capsici, Résistance induite, Nécrose,

Température.

\begin{abstract}
Des dosages de capsidiol ont été réalisés au cours de l'infection à différents niveaux dans les tiges contaminées. Ils montrent l'existence d'un gradient décroissant du haut vers le bas. Les concentrations sont maximales dans les tissus envahis et nécrosés et diminuent lorsqu'on s'en éloigne de manière approximativement exponenticlle. Une étude fine du phénomène semble indiquer que le gradient de capsidiol dans la tige s'établit plus en fonction de la position des tissus nécrosés que de celle des hyphes mycéliens.

L'existence de zones nécrotiques internes, particulièrement riches en capsidiol et se rencontrant préférentiellcment chez la varićté résistante «Phyo 636 " à $22^{\circ} \mathrm{C}$, introduit cependant une certaine hétérogénéité dans la distribution du capsidiol.

Il n'y a pas de relation entre la concentration en capsidiol et le degré de résistance induite aux différents niveaux de tiges infectées : il est suggéré que le capsidiol ne joue pas un rôle majeur dans l'expression de la résistance génétique du piment à $P$. capsici.
\end{abstract}

\section{SUMMARY}

\section{Capsidiol,}

Phytoalexin,

Capsicum annuum

Phytophthora capsici,

Induced resistance,

Necrosis,

Temperature.
Resistance of pepper (Capsicum annuum) to Phytophthora capsici

IX. Distribution of capsidiol in space and time in relation to induced resistance of infected stems

Capsidiol concentration was determined at different levels of pepper stems inoculated with Phytophthora capsici. A decreasing gradient from top to bottom was found. The concentration was maximum in the invaded necrotized tissues and decreased in a nearly exponential way as samples were taken lower and lower. A detailed study seemed to show that the capsidiol gradient was determined more by the localization of necrotized tissues than in relation to the advancing mycelial front.

With the resistant line «Phyo 636 » at $22^{\circ} \mathrm{C}$, the presence of internal necrotic zones containing high quantities of capsidiol entailed the heterogeneous distribution of the phytoalexin in the stems.

There was no correlation between the capsidiol concentration and the induced resistance scored at different levels of inoculated stems. Therefore, capsidiol seems to play only a minor role in the genetic resistance of pepper to $P$. capsici.

\section{INTRODUCTION}

La démonstration chez le piment d'une induction de résistance à Phytophthora capsici a pu être établie à la fois par des essais de recontamination (CLERJEAU \& NoURRISSEAU, 1976) et par la mise en évidence, au front de progression des hyphes, de substances inhibitrices de type phytoalexines, dont la plus importante en quantité est le capsidiol (Molot et al., 1976).

Au cours de l'infection, une étude cinétique des concentrations en capsidiol a pu être réalisée chez 4 variétés regroupées par couples quasi-isogéniques, sensibles ou résistants à $P$. capsici: d'une part "Yolo Wonder» et «Phyo 636 », d'autre part «Clairon» et « Fidelio ». Chez «Phyo 636 » et « Fidelio », la résistance dérive du même géniteur 493-1. Avec ce matériel, les dosages, toujours effectués au front de progression des hyphes du champignon, ont montré que la formation du capsidiol, plus précoce et plus intense chez les variétés résistantes que chez les variétés sensibles, atteint une valeur maximum au bout de $4 \mathrm{j}$ puis décroît par la suite (Molot et al., 1981).

Par ailleurs, dans un travail préliminaire (Molot et al., 1977), il avait été observé que :

- la distribution du capsidiol dans les tiges de piment contaminées par Phytophthora capsici semblait se faire à partir du point d'inoculation selon un gradient de concentrations décroissant ;

- cette répartition était cependant hétérogène, car il existait, au sein des tissus, des îlots bruns qui traduisaient une forte accumulation de capsidiol.

Dans la présente étude, nous nous proposons de vérifier de façon systématique ces observations en réalisant au cours de l'infection des dosages de capsidiol à différents niveaux des tiges contaminées (et non seulement au front de progression des hyphes). De plus, après avoir localisé visuellement la formation d'îlots nécrotiques selon la variété ( Yolo Wonder» ou "Phyo 636») et les conditions de contamination $\left(22^{\circ}\right.$ ou $\left.32^{\circ} \mathrm{C}\right)$, nous comparerons les taux de 
capsidiol dans les zones nécrotiques et non nécrotiques. Ces différentes données devraient apporter des éléments nouveaux sur le rôle du capsidiol dans la résistance des tiges de piment à $P$. capsici.

\section{MATÉRIEL ET MÉTHODES}

\section{A. Contamination du matériel végétal}

Au stade 7-8 feuilles, on décapite le sommet des tiges de piment, «Yolo Wonder» (YW) sensible et «Phyo 636 » résistant à $P$. capsici. Un fragment de culture mycélienne de

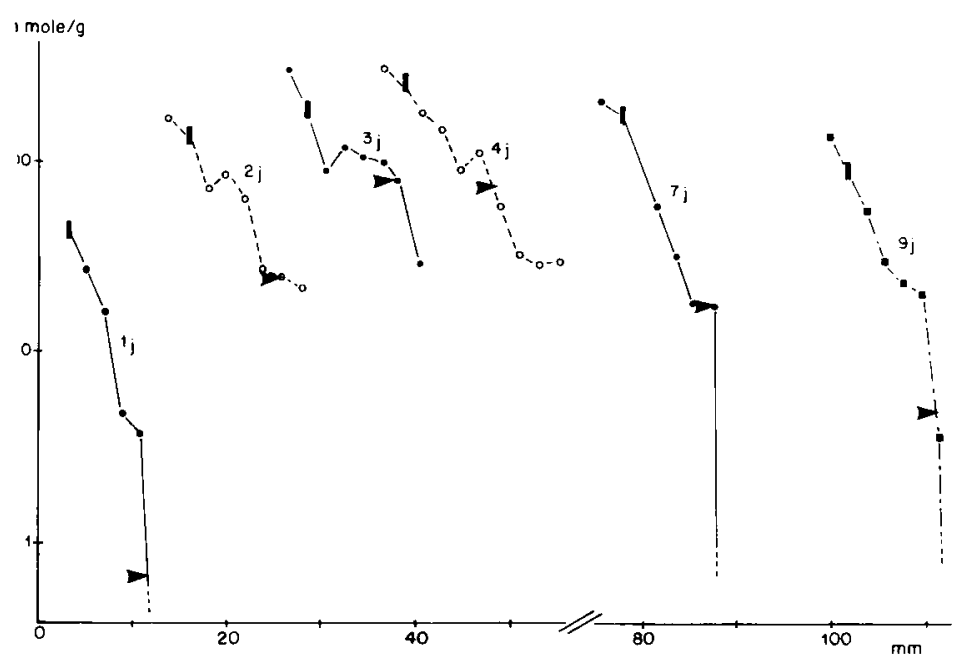

a)

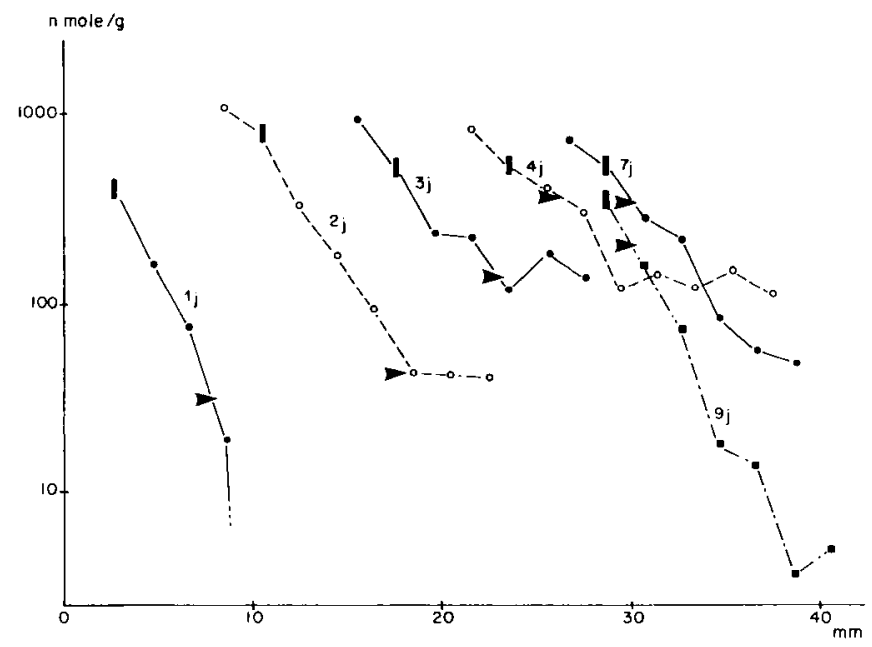

b)

Figure 1

Distribution du capsidiol dans les tiges de deux variétés de piment infectées par Phytophthora capsici : concentrations en n.moles/g de matière fraîche (échelle logarithmique) en fonction de la distance au point d'inoculation (en $\mathrm{mm}$ ) à différentes dates après l'inoculation. A chaque date, la barre et la flèche indiquent respectivement la limite inférieure de la nécrose externe et le front d'avance du mycélium. a) "Yolo Wonder ", b) "Phyo 636 ".

Distribution of capsidiol in the stems of two cultivars of pepper infected by Phytophthora capsici : concentrations in n.moles $/ g$ fresh weight (logarithmic scale) in relation to distance from the inoculation point (in $\mathrm{mm}$ ) at different times after inoculation. At each date, bar and arrow respectively indicate the lower limit of external necrosis and the advancing front of the mycelium. a) *Yolo Wonder *, b) * Phyo $636 »$. la souche monozoospore 15-12A est alors déposé sur la blessure selon une technique décrite par ailleurs (POCHARD et al., 1976). On portc les plantes en chambre climatisée, soit à 22 , soit à $32{ }^{\circ} \mathrm{C}$, à humidité relative $70 \mathrm{p} .100$. Au cours des jours qui suivent, il se développe le long de la tige une nécrose externe bien visible dont on peut mesurer la longueur.

\section{B. Prélèvement du matériel végétal en vue du dosage du capsidiol}

Des prélèvements sont effectués dans le temps (jusqu'à $9 \mathrm{j}$ après l'infection). Après avoir repéré sur chaque tige le front d'avance de la nécrose, on débite un $1^{\text {er }}$ tronçon, dit tronçon 0 , de $2 \mathrm{~mm}$ d'épaisseur, qui comporte autant de tissus nécrosés que non nécrosés. On poursuit la découpe de la tige soit vers le haut dans les tissus nécrosés extérieurement (tronçon-2), soit vers le bas dans les tissus non nécrosés extérieurement (tronçons $2,4,6$, etc...). Tous les tronçons ont $2 \mathrm{~mm}$ d'épaisseur. Les prélèvements correspondant à une date donnée sont effectués sur 20 plantes en moyenne et les tronçons d'un niveau déterminé sont regroupés entre eux.

Des isolements mycologiques permettent de situer la limite de progression des hyphes par rapport aux différents tronçons prélevés.

Pour un niveau donné (tronçons situés par exemple au front de progression des hyphes), on peut effectuer un tri visuel entre tronçons porteurs ou non de nécroses internes. On constitue ainsi 2 lots destinés à des dosages de capsidiol.

Les échantillons sont pesés, congelés, broyés dans l'éthanol à 50 p. 100 et, après évaporation de ce solvant, repris 3 fois par un égal volume d'éther. L'extrait évaporé à sec est alors redissous dans l'alcool. Les teneurs en capsidiol sont appréciées soit par chromatographie en phase gazeuse et exprimées alors en n.moles/g. de tissus frais (MOLOT et al., 1981), soit par chromatographie en couche mince. Dans ce dernicr cas, la migration s'effectue dans un mélange chloroforme-méthanol $(100-10 \mathrm{v} / \mathrm{v})$. Les chromatoplaques subissent une révélation biologique (MOLOT et al., 1976, 1980b) : le diamètre des zones d'inhibition de Cladosporium herbarum est proportionnel aux quantités de capsidiol. Des essais antérieurs (non publiés) ont montré une bonne corrélation entre les résultats des 2 méthodes chromatographiques dans une gamme comprise entre 10 et $1000 \mathrm{n}$.moles/g. de tissu frais.

\section{RÉSULTATS}

\section{A. Gradient de concentration en capsidiol clans les tiges infectées}

Les dosages effectués le long de tiges infectées (de $2 \mathrm{~mm}$ au-dessus de la limite de la nécrose externe jusqu'à 10 et $16 \mathrm{~mm}$ au-dessous), entre 1 et $9 \mathrm{j}$ après l'inoculation, révèlent l'existence, dans tous les cas, d'un gradient décroissant du haut vers le bas de la teneur des tissus en capsidiol (fig. 1). La concentration est maximale dans les tissus envahis et nécrosés (représentés par le tronçon- $2 \mathrm{~mm}$ ) et diminue, lorsqu'on s'en éloigne, de manière approximativement exponentielle. Les irrégularités observées, notamment chez « Phyo 636 » à 3 et $4 \mathrm{j}$, sont probablement attribuables à une répartition irrégulière des nécroses internes (cf. 
infra). On ne note pas de discontinuité dans le gradient à la limite externe de la nécrose ni au niveau du front de progression des hyphes.

L'évolution au cours du temps à ce dernier niveau a été décrite précédemment (MOLOT et al., 1981): chez ces 2 variétés, la concentration en capsidiol augmente rapidement jusque vers le $4^{\mathrm{e}} \mathrm{j}$ puis diminue, mais plus lentement, la variété résistante étant toujours plus riche en capsidiol. En revanche, on constate ici qu'à la limite inférieure de la nécrose externe, s'établit une teneur en capsidiol sensiblement constante entre le $2^{\mathrm{c}}$ et le $9^{\mathrm{e}} \mathrm{j}$ : en moyenne 180 n.moles $/ g$ chez « Yolo Wonder » et 560 n.moles $/ \mathrm{g}$. chez "Phyo 636 ".

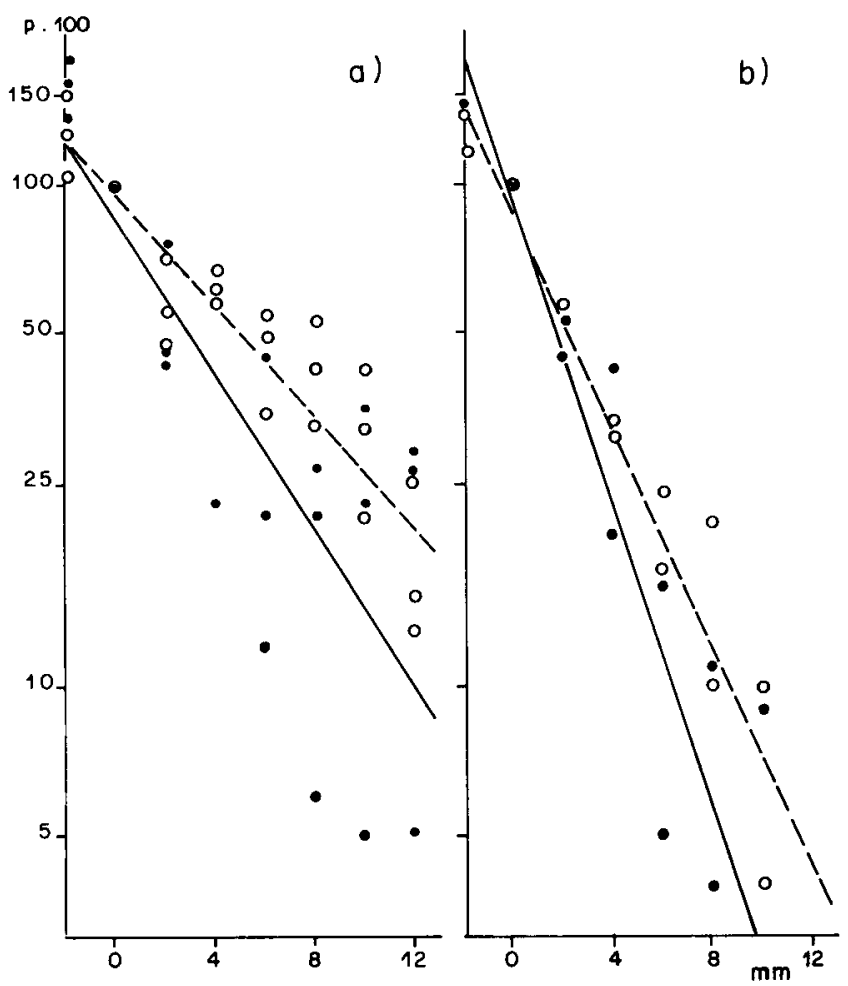

Figure 2

Forme des gradients de capsidiol par rapport à la limite inférieure de la nécrose externe (tronçon 0): concentrations en pourcentage de celle du tronçon 0 (échelle logarithmique) en fonction de la distance à la limite de la nécrose (en $\mathrm{mm}$ ). o....-o, "Yolo Wonder"; - - "Phyo 636". a) entre 2 et $4 j$, b) 7 et $9 j$ après inoculation.

Shape of capsidiol gradients from the lower limit of the external necrosis (section 0) : concentrations in percent of that in section 0 (logarithmic scale) in relation to distance from the necrosis limit (in mm) o.... o, "Yolo Wonder"; - "Phyo 636 ". a) between 2 and 4 days, b) 7 and 9 days after inoculation.

Calculés par rapport à ce niveau (fig. 2), les gradients ont une allure presque identique entre 2 et $4 \mathrm{j}$, avec toutefois une décroissance relative un peu plus rapide chez «Phyo $636 »$. Tout se passe comme si c'était le front de la nécrose et non celui du champignon qui commandait la répartition spatiale de la phytoalexine dans la tige. A 7 et 9 j, la décroissance se fait plus marquée. Chez « Phyo 636 », la nécrose est alors bloquée et, dans les zones situées à plus de $30 \mathrm{~mm}$ du point d'inoculation, on constate une diminution sur place de la teneur en capsidiol (fig. 1) ; il pourrait y avoir métabolisation de cette substance par les tissus demeurés sains.

\section{B. Mise en évidence de nécroses internes (tabl. 1)}

Chez la lignée «Phyo 636 », quand la résistance est bien établie ( $4 \mathrm{j}$ après la contamination), il est fréquent d'observer, au-delà de la zone profondément nécrosée, la présence d'îlots internes bruns répartis au hasard. Ceux-ci concernent principalement la zone vasculaire, mais aussi parfois les couches médullaires plus profondes. Ce brunissement conduit vraisemblablement à la mort des tissus et, par conséquent, à la formation d'une nécrose (MOLOT et al., 1977).

La présence de telles formations nécrotiques paraissant assez variable et quelques légères variations se manifestant dans le gradient des concentrations en capsidiol (fig. 1), nous avons entrepris une observation systématique des tronçons de tiges contaminées. Quatre jours après l'inoculation, nous avons séparé visuellement, niveau par niveau, les

\section{TABLEAU 1}

Pourcentage de tiges présentant des nécroses internes à différents niveaux du front de nécrose externe (4 jours après la contamination à $22^{\circ}$ ou $32^{\circ} \mathrm{C}$ )

Number (in percent) of stems with internal necrosis localized at different distances from the external necrosis limit (in $\mathrm{mm}$ ) 4 days afier inoculation, at $22^{\circ}$ or $32^{\circ} \mathrm{C}$

\begin{tabular}{|c|c|c|c|c|}
\hline \multirow{3}{*}{$\begin{array}{c}\text { Distance }(\mathrm{mm}) \\
\text { au front } \\
\text { de nécrose } \\
\text { externe }\end{array}$} & \multicolumn{4}{|c|}{ Contamination des tiges à : } \\
\hline & & & & \\
\hline & Y.W. & Phyo 636 & Y.W. & Phyo 636 \\
\hline 0 & 100 & 100 & 100 & 100 \\
\hline 2 & 8 & 91 & 3 & 32 \\
\hline 4 & 5 & 63 (a) & 2 & 16 \\
\hline 6 & 5 & 36 & 0 & $4(a)$ \\
\hline 8 & 4 & 32 & 0 & 0 \\
\hline 10 & 4 (a) & 20 & 0 & 0 \\
\hline 12 & 2 & 8 & 0 & 0 \\
\hline 14 & 0 & 6 & 0 (a) & 0 \\
\hline 16 & 1 & 6 & 0 & 0 \\
\hline 18 & 2 & 5 & 0 & 0 \\
\hline 20 & 1 & 5 & 0 & 0 \\
\hline 22 & 0 & 2 & 0 & 0 \\
\hline 24 & 0 & 3 & 0 & 0 \\
\hline 26 & 0 & 0 & 0 & 0 \\
\hline 28 & 0 & 3 & 0 & 0 \\
\hline 30 & 0 & 2 & 0 & 0 \\
\hline
\end{tabular}

a : front d'avance des hyphes

a: advancing front of the mycelium 
tronçons présentant des nécroses internes de ceux qui n'en présentent pas. Nous constatons que :

- les formations nécrotiques s'observent surtout chez la variété résistante, pratiquement pas chez la variété sensible ;

- elles sc manifestent avec une fréquence très élevéc quand les examens s'effectuent près des tissus infectés et nécrosés extérieurement (tronçon 0 ). Leur nombre diminue progressivement au fur et à mesure que l'on s'éloigne de ce site ;

- au-delà du front de progression des hyphes, chez la variété résistante, le pourcentage de plantes présentant des nécroses internes demeure élevé (de l'ordre de 35 p. 100) et il est encore possible d'observer quelques tronçons nécrotiques à $30 \mathrm{~mm}$ du front de nécrose externe ;

- dans le cas d'une contamination à $32^{\circ} \mathrm{C}$, on observe très peu de tronçons porteurs de nécroses internes. La progression des hyphes au bout de $4 \mathrm{j}$ est alors plus rapide qu'à $22^{\circ} \mathrm{C}$.

\section{Nécroses internes et capsidiol (tabl. 2)}

Deux ordres de phénomènes nous incitent à faire des rapprochements entre ces formations nécrotiques internes et les concentrations en capsidiol : d'une part, leur mode de répartition dans la tige (selon un gradient décroissant) et, d'autre part, leur absence à haute température. Des études antérieures ont, en effet, montré que la synthèse du capsidiol est pratiquement nulle chez «Yolo Wonder » et « Phyo 636 » à $32^{\circ} \mathrm{C}$ (Molot et al., 1977).

Afin de montrer d'une façon précise le lien existant entre ces 2 données, nous avons débité, 4 j après l'infection, des tiges de "Yolo Wonder» et de "Phyo 636" élevées à $22{ }^{\circ} \mathrm{C}$. Nous avons choisi 3 niveaux de prélèvement :

- tissus infectés, mais non nécrosés extérieurement: tronçons $n^{\circ} 4$;

- front de progression des hyphes : tronçons $n^{\circ} 12$ pour «Yolo Wonder », $n^{\circ} 6$ pour « Phyo $636 » ;$

- tissus non infectés : tronçons $\mathrm{n}^{\circ} 14$.

Chacun de ces niveaux a fait l'objet d'un tri visuel dans le but de séparer les tronçons présentant des nécroses de ceux n'en présentant pas. Des dosages de capsidiol ont été réalisés sur chacun de ces sous-ensembles (tabl. 2). Ils montrent que les tissus possédant des îlots nécrotiques sont toujours mieux pourvus en capsidiol (environ 2 fois plus chez « Yolo Wonder», de 2 à 7 fois plus chez « Phyo 636 »).

A la lumière de ces résultats, il apparaît que, si globablement les taux de capsidiol vont en diminuant à partir du point d'inoculation, la répartition de cettc phytoalexine est assez hétérogène et présente des pics de concentrations au niveau des îlots nécrotiques. Ce phénomène particulièrement net chez «Phyo 636 » peut également être observé chez «Yolo Wonder» (mais de façon moindre et incertaine).

\section{DISCUSSION}

Après contamination par $P$. capsici, il apparaît dans la tige de piment plusicurs phytoalexines; la plus abondante, le capsidiol, s'accumule en quantités d'autant plus importantes que les tissus prélevés sont plus proches du point d'inoculation. Nous avons pu observer de tels gradients pendant $9 \mathrm{j}$ après l'infection. Les 2 lignées de piment utilisées peuvent synthétiser cette substance, mais le matériel résistant en produit toujours davantage.

Cependant, chez la lignée «Phyo 636» (et également, mais de façon beaucoup moins nette, chez «Yolo Wonder $»$ ), des zones de nécroses internes se manifestent, à partir du $4^{\mathrm{e}} \mathrm{j}$, dans les tissus sains ou apparemment sains. Bien que leur répartition soit irrégulière, elles ont tendance à être d'autant moins fréquentes que l'on s'éloigne du site d'inoculation (jusqu'à $50-60 \mathrm{~mm}$ de ce dernier). Ces zones contiennent des quantités de capsidiol beaucoup plus importantes (de 2 à 7 fois plus) que les zones non nécrosées de même niveau. Leur existence perturbe donc les gradients de concentrations évoqués plus haut et traduit une hétérogénéité de distribution du capsidiol.

Ces zones de nécroses internes sont inexistantes à $32^{\circ} \mathrm{C}$, température qui bloque la synthèse du capsidiol (MOLOT et al., 1977). Un phénomène similaire a été signalé chez la pomme de terre infectée par Phytophthora infestans: l'élévation de température supprime le brunissement et bloque l'accumulation des terpenoïdes (LISKER \& KUC, 1978).

Pourquoi les zones de nécroses internes sont-elles toujours des secteurs privilégiés d'accumulation du capsidiol? Cette accumulation est-elle la cause ou la conséquence des brunissements observés? Nous ne possédons que peu d'informations relatives aux effets des phytoalexines sur les cellules des végétaux supérieurs. La pisatine est capable de réduire la croissance des racines du pois (CRUICKSHANK \& PERrIN, 1961); à la dose de $250 \mu \mathrm{g} / \mathrm{ml}$, cette même substance entraîne la lyse des protoplastes et le flétrissement des feuilles de pois (SHIRAISHI et al., 1975); ses effets s'exercent au niveau de la perméabilité et de la respiration

TABLEAU 2

Distribution du capsidiol (estimée par chromatographie en couche mince) dans les tiges de piment en fonction de la présence ou de l'absence de nécroses internes ( 4 jours après la contamination à $22^{\circ} \mathrm{C}$ )

Distribution of capsidiol (estimated by thin layer chromatography) in the pepper stem in presence or absence of internal necrosis (4 days after inoculation at $22^{\circ} \mathrm{C}$ )

\begin{tabular}{|c|c|c|c|c|c|c|}
\hline \multirow[t]{2}{*}{$\begin{array}{l}\text { Niveau de } \\
\text { prélèvement }\end{array}$} & \multicolumn{5}{|c|}{ Diamètre en $\mathrm{mm}$ de la zone d'inhibition avec: } & $1^{\circ} 14$ \\
\hline & Y.W. & Phyo 636 & Y.W. & Phyo 636 & Y.W. & Phyo 636 \\
\hline Avec nécroses internes & 21 & 42 & 7 & 25 & 4 & 21 \\
\hline Sans nécroses internes & 11 & 23 & 3 & 6 & 2 & 3 \\
\hline
\end{tabular}


des tissus (VAN ETTEN \& BATEMAN, 1971). Il en va de même pour la phaséolline dont les effets néfastes sur cellules en milieu liquide se manifestent à des concentrations encore plus faibles, de l'ordre de $50 \mu \mathrm{g} / \mathrm{ml}$ (SKIPP et al., 1977). Toutefois, STOEssL et al. (1977) ont montré que le capsidiol, injecté dans des fruits de piment, est au contraire rapidement métabolisé sans engendrer de phytotoxicité. Nous-mêmes avons observé ici, chez « Phyo 636 », que des tissus ayant contenu jusqu'à 300 n.moles de capsi$\mathrm{diol} / \mathrm{g}$ de tissu frais ne se nécrosent pas et voient ultérieurement leur teneur diminuer. On pourrait alors faire l'hypothèse que c'est le tissu nécrosé qui élicite dans le tissu sain environnant une biosynthèse accrue de capsidiol, selon un mécanisme analogue à celui décrit par HARGREAVES \& BAILEY (1978) chez le haricot. Cette vue paraît confortée par le fait que le gradient de capsidiol dans la tige s'établit en fonction de la position des tissus nécrosés plutôt que de celle des hyphes mycéliens.

Par rapport à « Yolo Wonder », la lignée résistante quasiisogénique «Phyo 636 » se caractérise, en réponse à l'infection par $P$. capsici, par une plus grande aptitude à former des nécroses et, corrélativement, à accumuler du capsidiol, mais aussi par un état plus élevé de résistance induite. Lorsque, $4 \mathrm{j}$ après inoculation, on recoupe la tige à une distance variable (jusqu'à $20 \mathrm{~mm}$ ) sous la limite externe de la nécrose et qu'on effectue une $2^{\mathrm{e}}$ inoculation, on constate une réduction de l'envahissement, par rapport à un témoin non pré-inoculé. Cette réduction est, en moyenne, constante aux différents niveaux, d'environ 14 p. $100 \mathrm{chez}$ «Yolo Wonder » et 90 p. 100 chez « Phyo 636 " (CLERJEAU \& NourrisseaU, 1976). Or, nous observons ici que, dans la même zone, la tencur en capsidiol décroît le long de la tige de 118 à 33 n.moles/g et de 330 à 119 n.moles/g respectivement chez ces 2 variétés et que, par ailleurs, la concentration la plus forte (330 n.moles/g) n'entraîne qu'une inhibition de 66 p. 100 de la croissance mycélienne in vitro de $P$. capsici (MOLOT et al., 1977 ; MOLOT et al., 1980a). Il ne semble donc pas y avoir de relation entre la concentration de cette phytoalexine et le degré de résistance induite aux différents niveaux des tiges infectées.

En vue d'interpréter correctement la relation capsidiolrésistance induite, nous poursuivons actuellement des expériences de double contamination à $22^{\circ}$ et $32^{\circ} \mathrm{C}$.

Reçu le 17 septembre 1981.
Accepté le 18 juin 1982.

\section{REMERCIEMENTS}

Les autcurs remercient vivement G. Rousse pour son importante contribution à la réalisation des dosages de capsidiol.

\section{RÉFÉRENCES BIBLIOGRAPHIQUES}

Clerjeau M., Nourrisseau J. G., 1976. La résistance du Piment à Phytophthora capsici. II. Variations de la réccptivité des tissus voisins de la zone infectéc, induites par la contamination. Ann. Phytopathol., 8, (4), 389-397.

Cruickshank A. M., Perrin D. R., 1961. Studies on phytoalexins. III. The isolation assay and general properties of a phytoalexin from Pisum sativum L. Austr. J. biol. Sci., 14, 336-348.

Hargreaves J. A., Bailey J. A., 1978. Phytoalexin production by hypocotyls of Phaseolus vulgaris in response to constitutive metabolites relcased by damaged bean cells. Physiol. Plant Pathol., 13, 89100.

Lisker N., Kuć J., 1978. Terpenoid accumulation and browning in potato sprouts inoculated with Phytophthora infestans. Phytopathology, 9, 68, 1284-1287.

Molot P. M., Clerjeau M., Nourrisseau J. G., Ricci P., 1976. La résistance du Piment (Capsicum annuum) à Phytophthora capsici. III. Etude sur extraits de tiges sensibles et résistantes du pouvoir antifongique induit par la contamination. Ann. Phytopathol., 8, (4), 399-409.

Molot P. M., Clerjeau M., Mas P., Ricci P., 1977. Rôle du Capsidiol dans la résistance génétique du Piment au Phytophthora capsici. C. R. $3^{e}$ cong., Eucarpia, Piment, Avignon-Montfavet, Juillet 77, 137-146.

Molot P. M., Mas P., Hilario A. L., 1980a. La résistance du Piment à Phytophthora capsici. VI. Pouvoir inhibiteur du Capsidiol sur la croissance et l'activité pectinolytique d'isolats d'agressivité variable. Ann. Phytopathol., 12, (1), 1-9.
Molot P. M., Mas P., Conus M., Ferrière H., 1980b. La résistance du Piment à Phytophthora capsici. VII. Protection des organes foliaires après mise en survie sur un filtrat de culture du parasite. Caractérisation des conditions d'expression du phénomène. Ann. Phytopathol, 12, (2), 95-107.

Molot P. M., Mas P., Conus M., Ferrière H., Ricci P., 1981. Relations between capsidiol concentration, spced of fungal invasion and level of induced resistance in cultivars of pepper (Capsicum annuum) susceptible or resistant to Phytophthora capsici. Physiol. Plant Pathol., 18, 379-389.

Pochard E., Clerjeau M., Pitrat M., 1976. La résistance du Piment au Phytophthora capsici. I. Mise en évidence d'une induction progressive de la résistance. Ann. Amélior. Plant., 28 (1), 35-50. Shiraishi T., Oku H., Isono M., Ouchi S., 1975. The injurious effect of pisation on the plasma membrane of Pea. Plant Cell Physiol., 16, 939-942.

Skipp R. A., Selby C., Bailey J. A., 1977. Toxic effects of phaseollin on plant cells. Physiol. Plant Pathol, 10, 221-227.

Stoessl A., Robinson J. R., Rock G. L., Ward E. W. B., 1977. Metabolism of capsidiol by sweet pepper tissue : some possible implications for phytoalexin studies. Phytopathology, 67, 64-66.

Van Etten H. D., Bateman D. F., 1971. Studies on the mode of action of the phytoalexin phaseollin. Phytopathology, 61, 13631372 . 